\title{
No Difference in Complication Rates or Patient- Reported Outcomes Between Bone-Patella Tendon-Bone and Quadriceps Tendon Autograft for Anterior Cruciate Ligament Reconstruction
}

\author{
Daniel W. Hogan, M.S., M. Benjamin Burch, M.D., Joseph M. Rund, M.D., \\ Derek W. Geeslin, B.S., Richard Ma, M.D., Aaron F. Gray, M.D., Constance R. Chu, M.D., \\ Taylor E. Ray, B.S., W. Michael Pullen, M.D., and Seth L. Sherman, M.D.
}

\begin{abstract}
Purpose: To compare subjective outcomes and complications of anterior cruciate ligament reconstruction (ACLR) using either bone-patellar tendon-bone (BPTB) or quadriceps tendon (QT) autograft. Methods: A retrospective analysis of prospectively collected data identified consecutive cohorts of patients undergoing ACLR with either BPTB or QT autograft. Patients with less than 12-month follow-up and those undergoing concomitant osteotomies, cartilage restoration, and/or other ligament reconstruction procedures were excluded. Pre- and postsurgical patient-reported outcomes including International Knee Documentation Committee, Knee Injury and Osteoarthritis Outcome Score, Patient-Reported Outcomes Measurement Information System (PROMIS), Single Assessment Numeric Evaluation, Tegner, and Marx were compared between groups. Complications requiring reoperation were recorded. Results: One hundred nineteen patients met inclusion criteria, including 39 QT autografts and 80 BPTB autografts. Demographic information was comparable between groups. Mean follow-up was comparable between groups (QT 22.4 \pm 10.6 months vs BPTB $28.5 \pm 18.5$ months, $P=.06$ ). At minimum 12 -month follow-up (range 12.0-100.8 months), patients in both groups demonstrated statistically significant improvements in International Knee Documentation Committee (QT 60.0\%, P<.0001; BPTB 57.7\%, P<.0001), all Knee Injury and Osteoarthritis Outcome Score domains, PROMIS Mobility T-Score (QT 27.2\%, $P=.0001$; BPTB 23.2\%, $P<.0001$ ), PROMIS Global Physical Health (QT $14.4 \%, P=.002$; BPTB 13.4\%, $P=.001$ ), PROMIS Physical Function (QT 29.6\%, $P<.0001 ;$ BPTB 37.1 \%,$P<.0001$ ), PROMIS Pain Interference $(\mathrm{QT}-16.5 \%, P<.0001$; BPTB $-20.8 \%, P<.0001)$, Single Assessment Numeric Evaluation, $(\mathrm{QT} 76.9 \%$, $P<.0001$; BPTB 73.3\%, $P<.0001$ ), Tegner (QT 92.9\%, $P=.0002$; BPTB $101.4 \%, P<.0001$ ), and Marx $(\mathrm{QT}-26.6 \%, P=.02$; BPTB $-32.0 \%, P=.0002)$ with no statistically significant differences between the 2 groups. Overall postoperative reoperation rate did not differ between groups (QT 12.8\% vs BPTB 23.8\%,P=.2). Revision ACL reconstruction rate did not differ between groups (QT 5.1\% vs BPTB 7.5\%,P=.6). Conclusions: Patients undergoing autograft ACLR with either BPTB or QT demonstrated significant subjective improvements in patient-reported outcomes from preoperative values and no statistically significant differences in outcomes between the groups. Complication and revision ACLR rates were similar between the 2 groups. Level of Evidence: III, retrospective cohort study.
\end{abstract}

A nterior cruciate ligament (ACL) injuries are one of the most common injuries of the knee for active individuals. Previous studies have estimated the

From the School of Medicine (D.W.H., D.W.G.) and Department of Orthopaedic Surgery (M.B.B., R.M., A.F.G.), University of Missouri, Columbia, Missouri; Department of Orthopaedic Surgery, University of Iowa, Iowa City, Iowa (J.M.R.); and Department of Orthopaedic Surgery, Stanford University, Palo Alto, California (C.R.C., T.E.R., W.M.P., S.L.S.), U.S.A.

The authors report the following potential conflicts of interest or sources of funding: R.A. reports grants from Cartiheal and Moximed, outside the submitted work. S.L.S. reports grants and personal fees from Arthrex; personal fees from BioVentus; personal fees and other from CONMED Linvatec; other from Epic Bio; personal fees from Flexion Therapeutics, GLG consulting, Joint Restoration Foundation, JRF Ortho, United States, Linvatec, Moximed, Olympus, Japan, RTI Surgical, United States, Smith $\theta$ Nephew, and Vericel; annual incidence of ACL injuries to be 60.9 per $100,000 .{ }^{1}$ Surgical techniques for anterior cruciate ligament reconstruction (ACLR) continue to evolve in

other from Vivorte; and personal fees from Zimmer, United States, outside the submitted work. Full ICMJE author disclosure forms are available for this article online, as supplementary material.

Received May 28, 2021; accepted October 22, 2021.

Address correspondence to Seth L. Sherman, M.D., 450 Broadway Pavilion

A, Redwood City, CA 94063.E-mail: shermans@stanford.edu

(C) 2021 THE AUTHORS. Published by Elsevier Inc. on behalf of the Arthroscopy Association of North America. This is an open access article under the CC BY-NC-ND license (http://creativecommons.org/licenses/by-nc-nd/4.0/).

2666-061X/21754

https://doi.org/10.1016/j.asmr.2021.10.019 
an effort to improve safety and efficacy, decrease complications, and decrease graft-site morbidity. Graft failure is an uncommon but devastating outcome. ACL reinjury occurs in approximately $3.6 \%$ of cases in patients who have undergone ACLR. ${ }^{2,3}$ Risk factors for reinjury have included younger patient age, graft type, and activity level. ${ }^{3,4}$ Ideal graft selection, an integral consideration for success of ACLR, remains controversial.

Each graft type possesses both positive and negative attributes. Bone-patellar tendon-bone (BPTB) autograft permits bone-to-bone fixation but carries an increased risk of postoperative anterior knee pain, difficulty kneeling, and patella fractures..$^{5-10}$ Hamstring tendon (HT) autograft has been shown in many studies to provide similar outcomes to BPTB autograft ${ }^{6-8,10-14}$; however, size can be unpredictable and with smaller grafts being associated with greater failure rates. ${ }^{15}$ In recent years, an alternative has increased in popularity and use-the quadriceps tendon (QT) autograft. Central QT autograft ACLR has previously been advocated by numerous authors. ${ }^{16-21}$ QT autografts have multiple potential benefits compared with BPTB and HT autografts, including preservation of hamstring anatomy and function, reduced incidence of anterior knee pain and numbness, decreased risk of patella fracture, and minimal bone bleeding. ${ }^{18}$ A historical concern for QT autograft was donor-site morbidity, including anterior knee pain, quadriceps muscle weakness, and decreased range of motion. ${ }^{22}$ Nonetheless, Mulford et al. $^{23}$ showed an anterior knee pain rate of only $3 \%$, full range of motion in $97 \%$ of patients and good quadriceps strength recovery. ${ }^{23}$ Moreover, when compared directly with BPTB and HT autograft, patients who received a QT autograft have been shown to have achieved knee extension sooner and required less pain medication after reconstruction. ${ }^{24}$ As such, it seems intuitive that QT represents a viable and reliable graft option with minimal donor-site morbidity. ${ }^{23}$

Despite favorable outcomes and a potentially reduced morbidity profile, the QT autograft is the least studied and least used autograft for ACLR. Polling data have demonstrated its use represents between $1 \%$ and $11 \%$ of all ACLR performed. ${ }^{25,26}$ Rather, BPTB autografts are the most used, often considered the gold standard for ACLR autograft, and are the benchmark to which other grafts are compared. ${ }^{2,4,27,28}$ There remains a lack of studies comparing outcomes of BPTB autografts and QT autografts.

The purpose of this study is to compare subjective outcomes and complications of ACLR using either BPTB or QT autograft. Our hypothesis is that there will be no difference in subjective outcome or complications between groups.

\section{Methods}

Following institutional review board approval (IRB \#2007099), a retrospective review of prospectively collected data identified consecutive cohorts of patients undergoing ACLR with either BPTB or QT autograft. Surgery was performed by a single fellowship-trained sports surgeon between 2011 and 2019. All patients were offered both graft choices as options. BPTB autografts were considered the "gold-standard" for contact athletes and used preferably in those athletes if no absolute or relative contraindications, including patella alta, patella baja or a history of Osgood-Schlatter disease. QT autografts were offered as an alternative graft choice after informed literature review and were preferred in young patients with high-athletic demand. All data, including demographics, primary and secondary diagnoses, and surgical details, were confirmed by the primary investigator. Patients undergoing concomitant osteotomies, cartilage restoration, and other ligament reconstruction procedures were excluded. All included patients had at least 12 months of postoperative follow-up. Patient reported outcomes were compiled using the software platform PatientIQ (Chicago, IL). Pre- and postsurgical patient-reported outcomes (PROs) including International Knee Documentation Committee (IKDC), Knee Injury and Osteoarthritis Outcome Score (KOOS-Pain, Symptoms, ADL, Sport, and Quality of Life), Patient-Reported Outcomes Measurement Information System (PROMIS), Single Assessment Numeric Evaluation (SANE), Tegner, and Marx were compared between groups. These PROs were used to quantify overall patient wellness, as well as knee function and pain levels. Complications requiring reoperation, including revision ACL reconstruction, arthrofibrosis, infection, pain, meniscus tears, cyclops lesions and patellar fractures, were recorded.

\section{Surgical Technique}

All procedures were performed with the patient under general anesthesia by a single fellowship-trained orthopaedic sports medicine surgeon. The ACL stump was meticulously debrided from the femoral and tibial footprints. The center of the tibial footprint was demarcated with anatomic landmarks, including the remnant anterior cruciate ligament stump, the anterior horn of the lateral meniscus, the medial and lateral tibial spines, and the intrameniscal ligament. The femoral center of the footprint, centered between the lateral intercondylar ridge and the posterior articular margin, was demarcated with a microfracture awl. Tunnels were drilled via independent technique using an anteromedial portal for the femoral tunnel and tip aiming tibial guide for the tibia. The femoral tunnel was visualized from both the anterolateral and anteromedial portal and determined to be in the accurate position. 
Table 1. Patient Demographics and Characteristics

\begin{tabular}{|c|c|c|c|c|c|c|}
\hline Graft & Age, y & Sex (\% Female $)$ & BMI & Height, cm & Ethnicity (\% Non-White) & Laterality (\% Left) \\
\hline$\overline{\mathrm{QT}}(\mathrm{N}=39)$ & $18.7 \pm 6.3$ & $42.1 \%$ & $25.9 \pm 7.7$ & $166.7 \pm 12.0$ & $17.9 \%$ & $46.2 \%$ \\
\hline ВРТВ $(\mathrm{N}=80)$ & $21.0 \pm 5.9$ & $42.5 \%$ & $26.8 \pm 5.6$ & $173.7 \pm 8.6$ & $23.8 \%$ & $48.1 \%$ \\
\hline$P$ value & .053 & 1.0 & .5 & $.0003^{*}$ & .5 & .8 \\
\hline
\end{tabular}

BMI, body mass index; BPTB, bone-patellar tendon-bone; QT, quadriceps tendon.

*Significance determined using $t$ test and $\chi^{2}$ test where appropriate; $P$ value less than or equal to .05 denotes significance.

The QT was harvested by using an approximately 6-cm longitudinal incision overlying the quadriceps tendon insertion. Patellar bone was not harvested for the QT grafts. Femoral and tibial fixation for the QT grafts were accomplished using the TightRope GraftLink technique (Arthrex, Inc., Naples, FL). For BPTB autograft, the central third of the patellar tendon was harvested along with bone from the patella and tibia at the respective tendon insertion sites. The harvested tissue was prepared on the back table. Femoral fixation was accomplished using either the adjustable suspensory technique with TightRope or traditional metallic screw technique. Fifty-one patients who received BPTB autograft underwent metallic screw fixation, whereas 29 had adjustable suspensory femoral fixation. Tibial fixation was accomplished using interference screw technique. Screw fixation was executed with titanium SOFTSILK screws (Smith \& Nephew, Andover, MA). Otherwise, the procedures were performed identically.

\section{Postoperative Protocol}

All patients underwent an identical postoperative protocol. Patients were discharged with detailed instructions for the postoperative plan, including aspirin deep vein thrombosis prophylaxis, foot pump exercises, narcotic pain medication, and a home-exercise program. Patients were fitted with a hinged knee brace, which was to be locked in extension and worn at all times for ambulation and for sleeping. Patients were instructed to be weight-bearing as tolerated with crutches to assist with walking. Home exercises were initiated 24 hours after surgery with the goal of complete extension and $90^{\circ}$ of flexion at initial follow-up, which was scheduled for 7 to 10 days postoperatively. Meniscus repair rehabilitation was the same in both groups with patients either weight bearing as tolerated or toe-touch weight-bearing based on their tear pattern. Formal physical therapy was initiated after the initial follow-up appointment. Return to greater-level activities were individualized, based on standardized, minimum time and progressive functional rehabilitation criteria.

\section{Statistical Analysis}

For each patient-reported outcome score and subscore, differences within and between the QT autograft cohort and the BPTB autograft cohort were analyzed.
Preoperative scores, >12-month postoperative scores, and comparisons between these scores were analyzed. Continuous distributed variables were analyzed using a Student $t$ test. Dichotomous data were analyzed using the Pearson $\chi^{2}$ test, and results were reported as a frequency and percentage. Microsoft Excel was used for statistical analysis (Microsoft, Inc., Redmond, WA). Post-hoc power analysis was generated in $G^{*}$ Power (Düsseldorf, Germany). ${ }^{29}$

\section{Results}

Current Procedural Terminology code "29888" identified 339 patients who underwent ACLR of any graft type between September 2011 and April 2019 with the primary surgeon. Fifty-one patients were excluded for undergoing ACLR with a graft type other than BPTB or QT. Of the remaining 288 patients, 97 patients were identified as undergoing QT autograft ACLR. Twentyfour of these 97 patients were excluded for not being a primary ACLR. Thirty-four additional QT ACLR patients were lost to follow-up or underwent concomitant osteotomy, cartilage restoration or other ligament reconstruction and were excluded. One-hundred ninety one patients were identified as undergoing BPTB ACLR. Of these 191 patients, 46 were identified as allografts and excluded. An additional 27 patients were excluded for not being a primary ACLR or due to concomitant osteotomy, cartilage restoration, or other ligament reconstruction. Of the remaining patients who underwent BTPB ACLR, only 80 patients met the 12month minimum follow-up requirement. A total of 119 patients met inclusion criteria, consisting of 39 QT and 80 BPTB autografts (Table 1). The mean age at time of surgery was 18.7 ( \pm 6.3 years) in the QT group and 21.0 ( \pm 5.9 years) in the BРTB group $(P=.053)$. Patient's mean height was $166.7 \mathrm{~cm}( \pm 12.0)$ in the QT group versus $173.7 \mathrm{~cm}( \pm 8.6)$ in the BPTB group $(P=$ $.0003)$. Sixteen of $39(41.0 \%)$ in the QT group and 34 of $80(42.5 \%)$ in the BPTB group were female $(P=$ 1.0). Mean body mass index was 25.9 in the QT group and 26.8 in the BPTB group $(P=.5)$. Seven of 39 $(17.9 \%)$ in the QT group and 19 of $80(23.8 \%)$ in the BPTB group were non-White $(P=.5)$. Sixteen of 39 $(41.0 \%)$ in the QT group and 42 of $80(52.5 \%)$ underwent concomitant meniscus repair $(P=.2)$.

PROs were listed for the QT and BPTB cohort, respectively (Table 2). There were no statistically 
Table 2. Preoperative and Postoperative Patient-Reported Outcomes

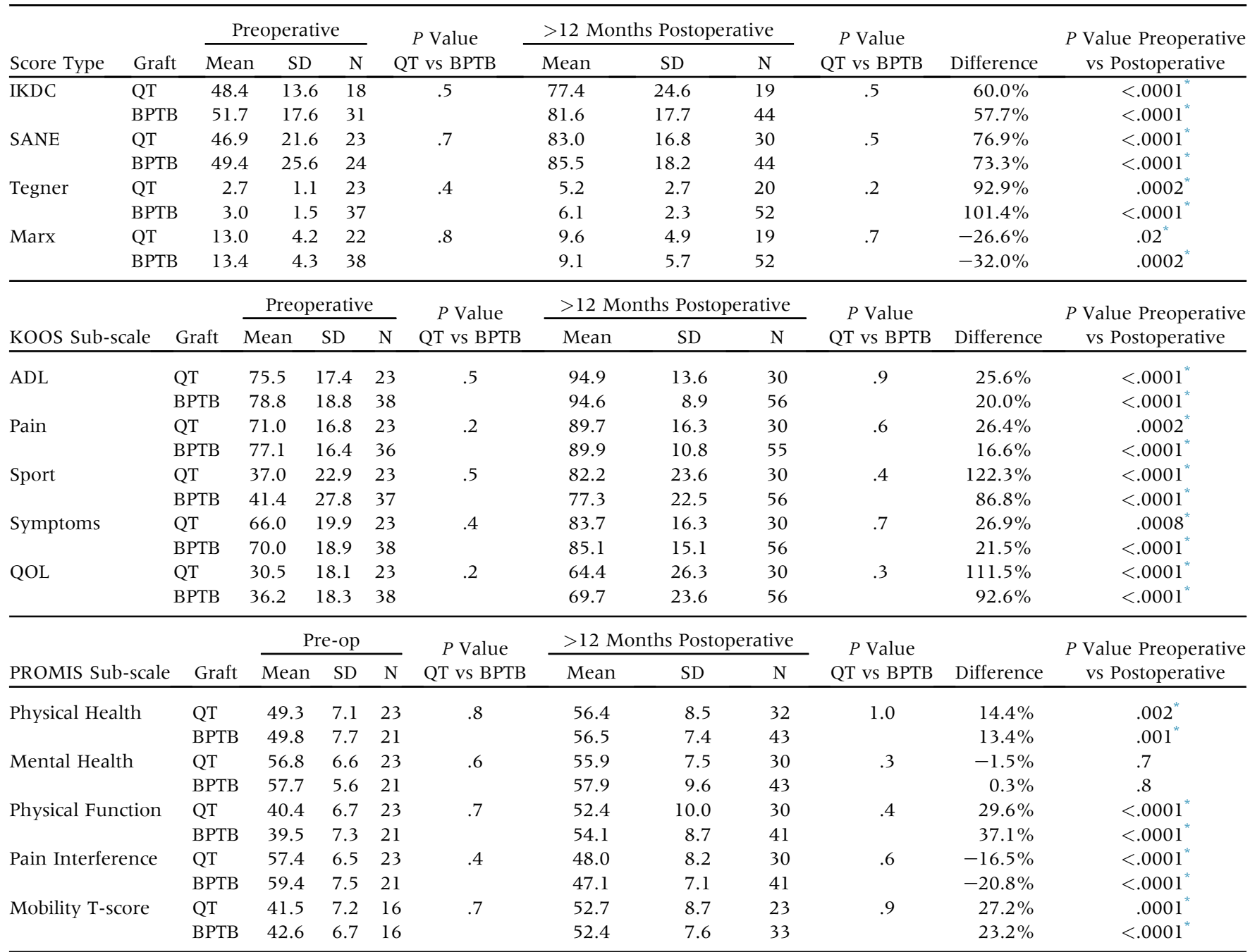

ADL, activities of daily living; BPTB, bone-patellar tendon-bone; IKDC, International Knee Documentation Committee; KOOS, Knee Injury and Osteoarthritis Outcome Score; PROMIS, Patient-Reported Outcomes Measurement Information System; QOL, quality of life; QT, quadriceps tendon; SANE, Single Assessment Numeric Evaluation; SD, standard deviation.

*Significance determined using the $t$ test and $\chi^{2}$ test where appropriate; $P$ value less than or equal to .05 denotes significance.

significant differences in baseline PROs, including IKDC, KOOS subsets, PROMIS scores, SANE, Tegner, and Marx.

At minimum 12-month follow up (range 12.0-100.8 months; QT $22.4 \pm 10.6$ months vs BPTB $28.5 \pm 18.5$ months, $P=.06$ ) patients in both QT and BPTB groups demonstrated statistically significant improvements from preoperative values in IKDC, all KOOS domains, PROMIS scores to include Mobility T, Global Physical Health, Physical Function, and Pain Interference, SANE scores, Tegner scores, and Marx scores (Table 2). Postoperative PROMIS Global Mental Health scores did not differ from preoperative scores in either group (QT $-1.5 \%, P=.7$; BPTB $0.3 \%$, $P=.9$ ). There were no differences in postoperative PROs between patients undergoing QT vs BPTB reconstruction (Table 2).
Complications were low and not statistically significant between groups (Table 3). The overall reoperation rate was $12.8 \%$ in the QT group compared with $23.8 \%$ in the BPTB group $(P=.2)$. The QT autograft group had 2 subsequent revision ACL reconstructions (5.1\%), and the BPTB group had 6 subsequent revision ACL reconstructions $(7.5 \%)(P=.6)$. Mean time to revision ACL reconstruction was 12.0 months (range 4.3-19.6 months) in the QT group and 25.2 months (range 3.772.4 months $)$ in the BPTB group $(P=.5)$. Mean time to any reoperation was 15.2 months (range 2.3-40.9 months) in the QT group and 21.4 months (range 0.597.8 months) in the BPTB group $(P=.7)$.

\section{Discussion}

In this comparative study, QT and BPTB autograft ACL reconstruction both demonstrated similar 
Table 3. Complications Requiring Reoperation in BPTB Autografts and QT Autografts

\begin{tabular}{|c|c|c|c|}
\hline Complications & BPTB Autograft $(\mathrm{N}=80)$ & QT Autograft $(\mathrm{N}=39)$ & $P$ Value \\
\hline Revision ACLR & $6(7.5 \%)$ & $2(5.1 \%)$ & .6 \\
\hline Arthrofibrosis & $6(7.5 \%)$ & $1(2.6 \%)$ & .3 \\
\hline Infection & $1(1.3 \%)$ & 0 & \\
\hline Meniscus tear & $2(2.5 \%)$ & $1(2.6 \%)$ & \\
\hline Cyclops lesion of ACL graft & $2(2.5 \%)$ & $1(2.6 \%)$ & \\
\hline Patella fracture & $1(1.3 \%)$ & 0 & \\
\hline
\end{tabular}

ACL, anterior cruciate ligament; ACLR, anterior cruciate ligament reconstruction; BPTB, bone-patellar tendon-bone; QT, quadriceps tendon. Significance determined using the $t$ test and $\chi^{2}$ test where appropriate; $P$ value less than or equal to .05 denotes significance.

postoperative improvement in patient report outcome measures with no statistically significant differences in reoperation rates. The results provide further support that QT autograft can produce reliable and similar results as compared with BPTB autograft reconstruction.

Objective physical examination measures have been studied following use of autograft ACLR. Previous studies have shown no difference in stability or knee range of motion following ACLR when comparing QT, HT, and BPTB autografts. ${ }^{30}$ Similarly, others have demonstrated no significant differences in knee laxity between HT and QT or BPTB and QT. ${ }^{31,32}$ A recent systematic review demonstrated no differences in postoperative Lachman, KT-1000, pivot shift, or range of motion when comparing graft types. ${ }^{33}$ Moreover, Mouarbes et al., ${ }^{34}$ in a meta-analysis of 27 clinical studies including 581 QT autografts, found no significant differences in Lachman test or pivot-shift test. Overall, these studies demonstrate no objective differences with range of motion or stability between graft types. $^{35}$

Most studies have demonstrated that no major differences exist in PROs, including subjective IKDC scores, KOOS Sport/Symptom scores, and Lysholm scores when comparing QT, BPTB, and HT graft choices. ${ }^{5,30-32,36-41}$ There is, however, heterogeneity in both graft fixation method and type of quadriceps graft harvested-quadriceps tendon with (QTB) or without (QT) bone block. Lund et al. ${ }^{32}$ randomized 51 patients to BPTB or QTB and found no difference in subjective IKDC scores and KOOS scores but showed decreased kneeling and graft-site pain with QTB. Similarly, Kim et al. $^{30}$ compared 27 BPTB autografts with 21 QTB autografts with minimum 2-year follow up and found no difference between the 2 groups in IKDC scores. Moreover, Han et al. ${ }^{37}$ compared 72 QTB autograft reconstructions with 72 BPTB autograft reconstructions with a minimum of 2-year follow up and found no difference between the groups in terms of stability, Lysholm scores, or IKDC scores. In contrast, Gorschewsky et al. ${ }^{36}$ followed 124 QTB ACL reconstructions with minimum 2-year follow up and compared them with a group of 136 BPTB autograft reconstructions. Their results favored BPTB on IKDC patient reported satisfaction and functional scores. These results, however, all used a proximal patellar bone block, which may have an impact on donor-site morbidity, postoperative pain, fixation, and outcomes. Our study compared soft tissue only QT with BPTB and similarly demonstrated no significant differences in postoperative outcome measures between these groups across all KOOS measures, IKDC, Tegner, SANE, and PROMIS measures.

Soft-tissue-only QT has seen a recent increase in the literature, with increased interest as fixation methods have changed. ${ }^{17,42}$ Early techniques used a hybrid of cortical suspensory fixation and interference screwfixation methods. ${ }^{18,19}$ While much of the literature has been focused on the biomechanical properties of the quadriceps graft, only a few recent studies have commented on PROs. ${ }^{17,42-45}$ Schulz et al. ${ }^{45}$ reported on a series of 55 QT ACLRs and demonstrated mean Lysholm scores of 89 , with $89.1 \%$ reporting good or very good results. They used bioabsorbable transfix pins for femoral and tibial fixation. Todor et al. ${ }^{44}$ compared 39 QT and 33 HT fixed with femoral cortical buttons and a tibial interference screw, identifying no differences in KT-1000 testing, Lysholm scores, modified Cincinnati, or the general SF-36. Our study, in contrast to those mentioned previously, reported outcome measures comparing BPTB with QT autografts. All patients improved from preoperative scores in both groups, and QT was not statistically different in outcome measures compared with BPTB autografts. The data presented in this study further support the use and study of soft-tissue-only QT as a viable and safe option for ACL reconstruction, performing comparable with BPTB in PRO measures at midterm follow-up. In addition, these data provide support for dual cortical suspensory fixation methods to secure our graft. Further long-term studies are needed to identify if these outcomes diminish over time.

Graft failure is also an important consideration when selecting a graft for ACL reconstruction. The majority of 
published data demonstrate that there are no differences in failure rates among the 3 most commonly used autografts. Hurley et al. ${ }^{33}$ demonstrated no differences in graft rerupture rates comparting QT and BPTB, with rates ranging from $0 \%$ to $2.8 \%$ for QT and $1.4 \%$ to $5.6 \%$ for BPTB. Similarly, comparing QTB and QT autografts, Crum et al. ${ }^{43}$ found no significant differences in graft rupture between groups, with results ranging from $0 \%$ to $9 \%$. Cavaignac et al. ${ }^{31}$ compared QT autografts with HT autografts and found a failure rate of $2.22 \%$ and $4.44 \%$, respectively. Younger age has consistently been associated with a greater rate of graft failure, with reports of $16.5 \%$ to $25 \%$ in patients younger than 25 years. ${ }^{3,46,47}$ Interestingly, in our study, the overall complication rate of the BPTB group $(23.8 \%)$ was almost double that of the QT group $(12.8 \%)$. Although not statistically significant, the large relative difference suggests the possibility of a type 2 error. In addition, our study demonstrated a revision ACL reconstruction rate of $5.1 \%$ in the QT autograft group and of $7.5 \%$ in the BPTB group, demonstrating no differences between the groups. Our median time to failure within the QT group was 12.0 months, suggesting that early fixation failure was not a significant issue using dual cortical suspensory fixation. Moreover, our study population also represented a very young population, mean 18.7 years QT and 21.0 years BPTB, with failure rates consistent with those within the reported literature. These results further support that QT is a safe, reliable, and effective graft for ACL reconstruction.

QT autografts offer potential anatomical and biomechanical advantages over alternative graft types. ${ }^{48}$ The mean cross-sectional area of the QT autograft has been shown to be significantly greater than that of the BPTB or HT autografts. ${ }^{49,50}$ This increased cross-sectional area may reduce the well-known bungee and windshield effects, as well as the tunnel-graft mismatch, which is postulated to cause inflow of synovial fluid and cytokines with subsequent bone resorption and tunnel widening. ${ }^{37,48,51}$ Moreover, knee strength should be considered when considering graft choice. A recent meta-analysis evaluating knee extensor strength found that QT knee extension strength was equivalent to BPTB with both weaker than HT. In addition, knee flexor strength was found to be greater in the QT autograft group than the HT autograft group. ${ }^{52}$

Our findings demonstrate good patient-reported functional and clinical outcomes and low complication rates at midterm follow-up following primary ACLR using QT autografts and BPTB autografts. Both groups showed significant improvements in almost all patient reported outcomes domains that we examined. Overall, the literature demonstrates that QT autografts show comparable clinical outcomes, functional outcomes and rupture rates compared with BPTB and HT autografts. ${ }^{34}$
As a result, QT autografts appear to be a reliable alternative to traditional grafts, such as the BPTB autograft. Surgeons should familiarize themselves with the advantages of each graft type to personalize the most effective treatment for each patient in consideration of the patient's age, activity level, occupation, and postoperative functional goals.

\section{Limitations}

We acknowledge many limitations to this study. First, graft choice was not randomized in these groups. The decision for graft type was made at the discretion of the surgeon after lengthy discussion with the patient. Second, as a single-surgeon study, there is a risk of performance bias. In addition, the data primarily consist of subjective questionnaires completed by patients rather than objective clinical measurements, which introduces possibility for error. Nonetheless, previous studies have demonstrated that patient reported outcomes are an important metric for understanding ACL reconstruction. ${ }^{53,54}$ Moreover, we feel that these limitations are mitigated by the fact that currently there is limited patient reported outcome data comparing BPTB to QT. Finally, the study is limited by the number of patients in both groups, which significantly limits the power. An estimated required sample size was calculated using the previously described minimal clinically important difference for KOOS of 8 points and a standard deviation of $15 .{ }^{55}$ In our study, with 80 patients in the BPTB group and 39 patients in the QT group, the group allocation ratio was approximately 2 . Using an effect size of $d=0.533$ at an allocation ratio of 2 , an estimated total sample size of 128 knees, including 85 BPTB knees and 43 QT knees, would be required to detect this difference at $80 \%$ power. $^{29}$ Although our groups approached the required sample size, a larger study may elucidate differences between the 2 groups.

\section{Conclusions}

Patients undergoing autograft ACLR with either BPTB or QT demonstrated significant subjective improvements in PROs from preoperative values and no statistically significant differences in outcomes between the groups. Complication and revision ACLR rates were similar between the 2 groups.

\section{References}

1. Parkkari J, Pasanen K, Mattila VM, Kannus P, Rimpelä A. The risk for a cruciate ligament injury of the knee in adolescents and young adults: A population-based cohort study of 46500 people with a 9 year follow-up. Br J Sports Med 2008;42:422-426.

2. Spindler KP, Kuhn JE, Freedman KB, Matthews CE, Dittus RS, Harrell FE. Anterior cruciate ligament reconstruction autograft choice: Bone-tendon-bone versus hamstring: Does it really matter? A systematic review. Am J Sports Med 2004;32:1986-1995. 
3. Pullen WM, Bryant B, Gaskill T, Sicignano N, Evans AM, DeMaio M. Predictors of revision surgery after anterior cruciate ligament reconstruction. Am J Sports Med 2016;44: 3140-3145.

4. Kaeding CC, Aros B, Pedroza A, et al. Allograft versus autograft anterior cruciate ligament reconstruction: Predictors of failure from a MOON prospective longitudinal cohort. Sports Health $2011 ; 3: 73-81$.

5. Geib TM, Shelton WR, Phelps RA, Clark L. Anterior cruciate ligament reconstruction using quadriceps tendon autograft: Intermediate-term outcome. Arthroscopy 2009;25:1408-1414.

6. Aune AK, Holm I, Risberg MA, Jensen HK, Steen H. Fourstrand hamstring tendon autograft compared with patellar tendon-bone autograft for anterior cruciate ligament reconstruction. A randomized study with two-year follow-up. Am J Sports Med 2001;29:722-728.

7. Ejerhed L, Kartus J, Sernert N, Köhler K, Karlsson J. Patellar tendon or semitendinosus tendon autografts for anterior cruciate ligament reconstruction? A prospective randomized study with a two-year follow-up. Am J Sports Med 2003;31:19-25.

8. Eriksson K, Anderberg P, Hamberg P, et al. A comparison of quadruple semitendinosus and patellar tendon grafts in reconstruction of the anterior cruciate ligament. J Bone Joint Surg Br 2001;83:348-354.

9. Feller JA, Webster KE. A randomized comparison of patellar tendon and hamstring tendon anterior cruciate ligament reconstruction. Am J Sports Med 2003;31: 564-573.

10. Laxdal G, Kartus J, Hansson L, Heidvall M, Ejerhed L, Karlsson J. A prospective randomized comparison of bone-patellar tendon-bone and hamstring grafts for anterior cruciate ligament reconstruction. Arthroscopy 2005;21:34-42.

11. Beard DJ, Anderson JL, Davies S, Price AJ, Dodd CA. Hamstrings vs. patella tendon for anterior cruciate ligament reconstruction: A randomised controlled trial. Knee 2001;8:45-50.

12. Jansson KA, Linko E, Sandelin J, Harilainen A. A prospective randomized study of patellar versus hamstring tendon autografts for anterior cruciate ligament reconstruction. Am J Sports Med 2003;31:12-18.

13. Shaieb MD, Kan DM, Chang SK, Marumoto JM, Richardson AB. A prospective randomized comparison of patellar tendon versus semitendinosus and gracilis tendon autografts for anterior cruciate ligament reconstruction. Am J Sports Med 2002;30:214-220.

14. Webster KE, Feller JA, Hameister KA. Bone tunnel enlargement following anterior cruciate ligament reconstruction: A randomised comparison of hamstring and patellar tendon grafts with 2-year follow-up. Knee Surg Sports Traumatol Arthrosc 2001;9:86-91.

15. Conte EJ, Hyatt AE, Gatt CJ Jr, Dhawan A. Hamstring autograft size can be predicted and is a potential risk factor for anterior cruciate ligament reconstruction failure. Arthroscopy 2014;30:882-890.

16. Mehran N, Damodar D, Shu Yang J. Quadriceps tendon autograft in anterior cruciate ligament reconstruction. J Am Acad Orthop Surg 2020;28:45-52.
17. Sheean AJ, Musahl V, Slone HS, et al. Quadriceps tendon autograft for arthroscopic knee ligament reconstruction: Use it now, use it often. Br J Sports Med 2018;52:698-701.

18. Fulkerson JP. Central quadriceps free tendon for anterior cruciate ligament reconstruction. Op Tech Sports Med 1999;7:195-200.

19. Fulkerson JP, Langeland R. An alternative cruciate reconstruction graft: The central quadriceps tendon. Arthroscopy 1995; 1 1:252-254.

20. Stäubli HU. Arthroscopically assisted ACL reconstruction using autologous quadriceps tendon. In: Jakob RP, Stäubli HU, eds. The knee and the cruciate ligaments. Berlin, Heidelberg: Springer, 1992.

21. Blauth W. 2-strip substitution-plasty of the anterior cruciate ligament with the quadriceps tendon. Unfallheilkunde 1984;87:45-51 [in German].

22. West RV, Harner CD. Graft selection in anterior cruciate ligament reconstruction. J Am Acad Orthop Surg 2005;13: 197-207.

23. Mulford JS, Hutchinson SE, Hang JR. Outcomes for primary anterior cruciate reconstruction with the quadriceps autograft: A systematic review. Knee Surg Sports Traumatol Arthrosc 2013;21:1882-1888.

24. Joseph M, Fulkerson J, Nissen C, Sheehan TJ. Short-term recovery after anterior cruciate ligament reconstruction: A prospective comparison of three autografts. Orthopedics 2006;29:243-248.

25. Middleton KK, Hamilton T, Irrgang JJ, Karlsson J, Harner CD, Fu FH. Anatomic anterior cruciate ligament (ACL) reconstruction: A global perspective. Part 1. Knee Surg Sports Traumatol Arthrosc 2014;22:1467-1482.

26. van Eck CF, Illingworth KD, Fu FH. Quadriceps tendon: The forgotten graft. Arthroscopy 2010;26:441-442. author reply 442-443.

27. Magnussen RA, Carey JL, Spindler KP. Does autograft choice determine intermediate-term outcome of ACL reconstruction? Knee Surg Sports Traumatol Arthrosc $2011 ; 19: 462-472$.

28. Carmichael JR, Cross MJ. Why bone-patella tendon-bone grafts should still be considered the gold standard for anterior cruciate ligament reconstruction. Br J Sports Med 2009;43:323-325.

29. Faul F, Erdfelder E, Buchner A, Lang AG. Statistical power analyses using $G^{*}$ Power 3.1: Tests for correlation and regression analyses. Behav Res Methods 2009;41: $1149-1160$.

30. Kim S-J, Kumar P, Oh K-S. Anterior cruciate ligament reconstruction: Autogenous quadriceps tendon-bone compared with bone-patellar tendon-bone grafts at 2year follow-up. Arthroscopy 2009;25:137-144.

31. Cavaignac E, Coulin B, Tscholl P, Nik Mohd Fatmy N, Duthon V, Menetrey J. Is quadriceps tendon autograft a better choice than hamstring autograft for anterior cruciate ligament reconstruction? A comparative study with a mean follow-up of 3.6 years. Am J Sports Med 2017;45: 1326-1332.

32. Lund B, Nielsen T, Faunø P, Christiansen SE, Lind M. Is quadriceps tendon a better graft choice than patellar tendon? A prospective randomized study. Arthroscopy 2014;30:593-598. 
33. Hurley ET, Calvo-Gurry M, Withers D, Farrington SK, Moran R, Moran CJ. Quadriceps tendon autograft in anterior cruciate ligament reconstruction: A systematic review. Arthroscopy 2018;34:1690-1698.

34. Mouarbes D, Menetrey J, Marot V, Courtot L, Berard E, Cavaignac E. Anterior cruciate ligament reconstruction: A systematic review and meta-analysis of outcomes for quadriceps tendon autograft versus bone-patellar tendon-bone and hamstring-tendon autografts. Am J Sports Med 2019;47:3531-3540.

35. Renström PA. Eight clinical conundrums relating to anterior cruciate ligament (ACL) injury in sport: Recent evidence and a personal reflection. Br J Sports Med 2013;47:367-372.

36. Gorschewsky O, Klakow A, Pütz A, Mahn H, Neumann W. Clinical comparison of the autologous quadriceps tendon (BQT) and the autologous patella tendon (ВРТВ) for the reconstruction of the anterior cruciate ligament. Knee Surg Sports Traumatol Arthrosc 2007;15:1284-1292.

37. Han HS, Seong SC, Lee S, Lee MC. Anterior cruciate ligament reconstruction: Quadriceps versus patellar autograft. Clin Orthop Relat Res 2008;466:198-204.

38. Runer A, Csapo R, Hepperger C, Herbort M, Hoser C, Fink C. Anterior cruciate ligament reconstructions with quadriceps tendon autograft result in lower graft rupture rates but similar patient-reported outcomes as compared with hamstring tendon autograft: A comparison of 875 patients. Am J Sports Med 2020;48:2195-2204.

39. Fischer F, Fink C, Herbst E, et al. Higher hamstring-toquadriceps isokinetic strength ratio during the first postoperative months in patients with quadriceps tendon compared to hamstring tendon graft following ACL reconstruction. Knee Surg Sports Traumatol Arthrosc 2018;26:418-425.

40. Lee S, Seong SC, Jo H, Park YK, Lee MC. Outcome of anterior cruciate ligament reconstruction using quadriceps tendon autograft. Arthroscopy 2004;20: 795-802.

41. Sofu H, Sahin V, Gürsu S, Yildırım T, Issin A, Ordueri M. Use of quadriceps tendon versus hamstring tendon autograft for arthroscopic anterior cruciate ligament reconstruction: A comparative analysis of clinical results. Eklem Hastalik Cerrahisi 2013;24:139-143.

42. Slone HS, Romine SE, Premkumar A, Xerogeanes JW. Quadriceps tendon autograft for anterior cruciate ligament reconstruction: A comprehensive review of current literature and systematic review of clinical results. Arthroscopy 2015;31:541-554.

43. Crum RJ, Kay J, Lesniak BP, Getgood A, Musahl V, de Sa D. Bone versus all soft tissue quadriceps tendon autografts for anterior cruciate ligament reconstruction: A systematic review. Arthroscopy 2020;37:1040-1052.
44. Todor A, Nistor DV, Caterev S. Clinical outcomes after ACL reconstruction with free quadriceps tendon autograft versus hamstring tendons autograft. A retrospective study with a minimal follow-up two years. Acta Orthop Traumatol Turc 2019;53:180-183.

45. Schulz AP, Lange V, Gille J, et al. Anterior cruciate ligament reconstruction using bone plug-free quadriceps tendon autograft: Intermediate-term clinical outcome after 24-36 months. Open Access J Sports Med 2013;4: 243-249.

46. Kamien PM, Hydrick JM, Replogle WH, Go LT, Barrett GR. Age, graft size, and Tegner activity level as predictors of failure in anterior cruciate ligament reconstruction with hamstring autograft. Am J Sports Med 2013;41:1808-1812.

47. Barrett AM, Craft JA, Replogle WH, Hydrick JM, Barrett GR. Anterior cruciate ligament graft failure: A comparison of graft type based on age and Tegner activity level. Am J Sports Med 201 1;39:2194-2198.

48. Runer A, Wierer G, Herbst E, et al. There is no difference between quadriceps- and hamstring tendon autografts in primary anterior cruciate ligament reconstruction: A 2year patient-reported outcome study. Knee Surg Sports Traumatol Arthrosc 2018;26:605-614.

49. Stäubli HU, Schatzmann L, Brunner P, Rincón L, Nolte LP. Mechanical tensile properties of the quadriceps tendon and patellar ligament in young adults. Am J Sports Med 1999;27:27-34.

50. Hamner DL, Brown CH, Steiner ME, Hecker AT, Hayes WC. Hamstring tendon grafts for reconstruction of the anterior cruciate ligament: Biomechanical evaluation of the use of multiple strands and tensioning techniques. J Bone Joint Surg Am 1999;81:549-557.

51. Paessler HH, Mastrokalos DS. Anterior cruciate ligament reconstruction using semitendinosus and gracilis tendons, bone patellar tendon, or quadriceps tendon-graft with press-fit fixation without hardware: A new and innovative procedure. Orthop Clin North Am 2003;34:49-64.

52. Johnston PT, McClelland JA, Feller JA, Webster KE. Knee muscle strength after quadriceps tendon autograft anterior cruciate ligament reconstruction: Systematic review and meta-analysis. Knee Surg Sports Traumatol Arthrosc 2021;29:2918-2933.

53. Herrington L. Functional outcome from anterior cruciate ligament surgery: A review. OA Orthop 2013;1:12-19.

54. Kocher MS, Steadman JR, Briggs KK, Sterett WI, Hawkins RJ. Relationships between objective assessment of ligament stability and subjective assessment of symptoms and function after anterior cruciate ligament reconstruction. Am J Sports Med 2004;32:629-634.

55. Roos EM, Lohmander LS. The Knee injury and Osteoarthritis Outcome Score (KOOS): From joint injury to osteoarthritis. Health Qual Life Outcome 2003;1:64. 